\title{
Systems Biology: A Therapeutic Target for Tumor Therapy
}

\author{
Albrecht Reichle $\cdot$ Thomas Vogt
}

Received: 28 April 2008 / Accepted: 26 June 2008 / Published online: 23 July 2008

(C) The Author(s) 2008

\begin{abstract}
Tumor-related activities that seem to be operationally induced by the division of function, such as inflammation, neoangiogenesis, Warburg effect, immune response, extracellular matrix remodeling, cell proliferation rate, apoptosis, coagulation effects, present itself from a systems perspective as an enhancement of complexity. We hypothesized, that tumor systems-directed therapies might have the capability to use aggregated action effects, as adjustable sizes to therapeutically modulate the tumor systems' stability, homeostasis, and robustness. We performed a retrospective analysis of recently published data on 224 patients with advanced and heavily pre-treated $(10 \%$ to $63 \%$ ) vascular sarcoma, melanoma, renal clear cell, cholangiocellular, carcinoma, hormone-refractory prostate cancer, and multivisceral Langerhans' cell histiocytosis enrolled in nine multi-center phase II trials (11 centers). Each patient received a multi-targeted systems-directed therapy that consisted of metronomic low-dose chemotherapy, a COX-2 inhibitor, combined with one or two transcription modulators, pioglitazone $+/-$ dexamethasone or IFN-alpha. These treatment schedules may attenuate the metastatic potential, tumor-associated inflammation, may exert site-specific activities, and induce long-term disease stabilization followed by prolonged objective response (3\% to $48 \%$ ) despite poor monoactivity of the respective drugs.
\end{abstract}

\footnotetext{
A. Reichle $(\triangle)$

Department of Hematology and Oncology,

University Hospital of Regensburg,

93042 Regensburg, Germany

e-mail: albrecht.reichle@klinik.uni-regensburg.de

T. Vogt

Department of Dermatology, University Hospital Regensburg,

Regensburg, Germany
}

Progression-free survival data are comparable with those of reductionist-designed standard first-line therapies. The differential response patterns indicate the therapies' systems biological activity. Understanding systems biology as adjustable size may break through the barrier of complex tumor-stroma-interactions in a therapeutically relevant way: Comparatively high efficacy at moderate toxicity. Structured systems-directed therapies in metastatic cancer may get a source for detecting the topology of tumor-associated complex aggregated action effects as adjustable sizes available for targeted biomodulatory therapies.

Keywords Systems biology · Tumor microenvironment . Transcription factors · Pioglitazone $\cdot$ PPARs .

Dexamethasone $\cdot$ Interferon-alpha $\cdot \mathrm{COX}-2$ inhibitor .

Metronomic low dose chemotherapy

\section{Introduction}

Unlike laws of nature, causal relations between initiating processes of tumor development are not anchored in an invariance of nature. Therefore, molecular and cytogenetic aberrations at initial diagnosis are generally heterogeneous in both tumors and single tumor types. Invariance within the tumor process may be observed during tumor progression. In interaction with normal human tissue, tumor cells use processes according to laws of nature to build up a favorable infrastructure for proliferation. In 1986, Dvorak interpreted for the first time these laws of nature as tumor-associated 'wound healing' mechanisms such as angiogenesis, inflammation, immunology, remodeling of the extracellular matrix, specific changes in cell metabolism and coagulation, and altered behavior in proliferation [1-7]. Accordingly, tumors may be figuratively conceived as 'never healing wounds'. With this 
interpretation, Dvorak addressed the systems biology of a tumor in a contemporary context. Up to now, a tumor's systems biology has rarely presented a target for a systematic approach in cancer treatment.

The dysregulated systems biology of a tumor may commonly not be understood mono-causally or explained context-free. The tumor's systems biology intents on a dysbalance between interfering functional elements in a way that conditioning and conditioned tumor-promoting elements (e.g. wound healing mechanisms) behave reciprocally also under therapeutic aspects.

The dysregulation of wound healing mechanisms is reflected in tumor-associated disease traits (e.g. tumorassociated inflammation, ECOG performance status, thrombophilia, and tumor-associated auto-immunity) and on the molecular level in the dysregulation of (nuclear) transcription factors, both in tumor and neighboring stroma cells. Transcription factors regulate in a concerted action distinct gene cascades and consecutively important cell functions for survival. Their cooperative interaction is also important for the survival of tumor cells.

In seven published phase II trials, we combined modulators (ligands) of (nuclear) transcription factors (pioglitazone, dexamethasone, interferon-alpha, cyclo-oxygenase-2 (COX-2) inhibitors) with the aim to suppress tumorassociated inflammation [8-15]. Corticosteroids are known for their anti-inflammatory activity; interferon-alpha at low doses (3.0 to 4.5 MU three times a week) shows both antiinflammatory and angiostatic activity as well as the antidiabetic drug pioglitazone (peroxisome proliferator-activated receptor (PPAR)-alpha/gamma agonist) [16-18]. Besides its anti-inflammatory activity, the COX-2 inhibitor also exerts an anti-proliferative via suppression of the PPAR-delta expression [19]. The efficacy of the anti-inflammatory therapy approach was controlled by the measurement of Creactive protein (CRP) levels in serum.

To enhance the therapeutic efficacy, a second wound healing mechanism was therapeutically targeted: neoangiogenesis. Metronomic low-dose chemotherapy with either trofosfamide or capecitabine may enhance the important antiangiogenic factor thrombospondin-1 in serum with simultaneously negligible cytotoxic activity of the respective drugs [20]. The present therapeutic approach - a combination of anti-inflammatory, angiostatic and immunomodulatory therapy - is primarily directed against invariant mechanisms embedded in the laws of nature that are generally important during tumor progression. Therefore, treatment efficacy may be expected to some degree, independently of the tumor type.

The summary of recently published data on combined anti-inflammatory and angiostatic therapy approaches in metastatic cancer may support the 'wound healing' hypothesis from a therapeutic view. Firstly, we want to show with our data from seven clinical trials that different antiinflammatory approaches are not only clinically efficacious and safe but show a moderate toxicity profile and may even induce continuous complete remission in combination with angiostatic therapies. Secondly, we are going to demonstrate according to the observed typical response characteristics that our therapeutic approaches have primarily biomodulatory rather than classic cytotoxic activity. Thirdly, we have introduced combined anti-inflammatory and angiostatic approaches for the therapy of metastatic tumors. The combined activity may even induce continuous complete remission.

The summarized results of the presented biomodulatory therapy approaches in different metastatic tumors contradict the paradigm that for the most part only drug-mediated blockades of more or less tumor-specific aberrant pathways may induce tumor response, a paradigm which is supported by an overwhelming number of clinical data.

\section{Patients and Methods}

\section{Selection of Metastatic Diseases}

We performed retrospective analyses of recently published data from our study group on patients with advanced and heavily pre-treated tumors (Table 1). According to our chosen therapeutic approaches - a combined anti-inflammatory and angiostatic therapy - we selected (1) tumors with high vascular density such as vascular sarcomas and renal clear cell carcinomas (RCCC), (2) a highly inflammatory tumor type, i.e. chemo-resistant multivisceral Langerhans' cell histiocytosis, and (3) tumors with a known inflammatory component at least in the metastatic stage (melanoma, cholangiocellular carcinoma, and hormone-refractory prostate cancer (HRPC). All patients were enrolled in phase II trials, and melanoma patients additionally participated in a randomized phase II trial.

\section{Patients' Characteristics}

The local ethics committee approved study protocols, and patients were required to provide written informed consent before enrolment. Patients presented were recruited between February 2001 and July 2006 in seven phase II trials including one randomized phase II trial in metastatic melanoma. Patients with advanced bidimensionally measurable neoplasias, either systemically pretreated or not, who experienced disease progression and who had a life expectancy of more than three months were eligible for the studies. Controlled brain metastases were no exclusion criteria. The remaining inclusion criteria are indicated in the respective publication. 
Table 1 Combined targeting of (nuclear) transcription factors

\begin{tabular}{|c|c|c|c|c|c|c|c|}
\hline \multirow[t]{2}{*}{ Tumor type } & \multirow{2}{*}{$\begin{array}{l}\text { Metronomic } \\
\text { Chemotherapy }\end{array}$} & \multirow{2}{*}{$\begin{array}{l}\text { No. of } \\
\text { patients }\end{array}$} & \multicolumn{4}{|c|}{ Receptor agonist/antagonist } & \multirow[t]{2}{*}{ Publications } \\
\hline & & & $\begin{array}{l}\text { PPAR } \alpha / \gamma \\
\text { agonist }^{\mathrm{a}}\end{array}$ & $\begin{array}{l}\text { PPAR } \delta \\
\text { antagonist }^{b}\end{array}$ & Glucocorticoid $^{\mathrm{c}}$ & IFN- $\alpha^{\mathrm{d}}$ & \\
\hline Kaposi sarcoma & Trofosfamide & 1 & + & + & - & - & Arch Dermatol, 2004 \\
\hline Angiosarcomas & Trofosfamide & 6 & + & + & - & - & Cancer, 2003 \\
\hline Sarcomas I & Trofosfamide & 21 & + & + & - & - & Cancer, 2004 \\
\hline Melanoma I & Trofosfamide & 19 & + & + & - & - & Cancer, 2004 \\
\hline Melanoma II Arm A & & 35 & - & - & - & - & Melanoma Research, 2007 \\
\hline Arm B & & 32 & + & + & - & - & \\
\hline Langerhans' cell histiocytosis & Trofosfamide & 2 & + & + & - & - & Br. J.Haematol, 2005 \\
\hline Renal clear cell carcinoma I & Capecitabine & 18 & + & + & - & - & Biomarker Insights, 2006 \\
\hline $\begin{array}{l}\text { Renal clear cell } \\
\text { carinoma II }\end{array}$ & Capecitabine & 33 & + & + & - & + & Biomarker Insights, 2006 \\
\hline $\begin{array}{l}\text { Hormone-refractory } \\
\text { prostate cancer }\end{array}$ & Capecitabine & 36 & + & + & + & - & $\begin{array}{c}\text { Lancet Oncology, } 2006 \\
\text { ASCO abstract, } 2007\end{array}$ \\
\hline $\begin{array}{l}\text { Cholangiocellular } \\
\text { carcinoma }\end{array}$ & Capecitabine & 21 & + & + & - & - & $\begin{array}{l}\text { Tumor Microenvironment } \\
\text { Prague, } 2004 \text { (Medimond) }\end{array}$ \\
\hline
\end{tabular}

PPAR peroxisome proliferator-activated receptor

a Pioglitazone

${ }^{\mathrm{b}}$ Selective COX-2 inhibitor

c dexamethasone

$\mathrm{d}$ interferon- $\alpha$

\section{Basic Treatment Considerations}

Treatment schedules were intended to achieve disease stabilization in metastatic neoplasias of different origin with uniform biomodulatory treatment principles and to limit therapy-related toxicity in advanced tumor stages. All patients received a combined anti-inflammatory and angiostatic therapy consisting of (1) metronomic low-dose chemotherapy (trofosfamide or capecitabine), (2) COX-2/ PPAR (peroxisome proliferator-activated receptor)-delta blockade (rofecoxib or etoricoxib) combined with (3) one or two transcription modulators, i.e. pioglitazone (peroxisome proliferator receptor alpha/gamma agonist) +/dexamethason or pioglitazone $+/-$ IFN-alpha (Table 1) [8-15].

\section{Anti-inflammatory Therapies}

We have chosen drugs with transcriptional activity in the field of inflammation control: glucocorticoids (dexamethasone 0.5 to $1.0 \mathrm{mg}$ daily), interferon-alpha (3 to $4.5 \mathrm{MU}$ three times a week), and the glitazone pioglitazone (45 to $60 \mathrm{mg}$ daily). Also the administered coxibs (rofecoxib 12.5 to $25 \mathrm{mg}$ daily or etoricoxib $60 \mathrm{mg}$ daily) may express transcriptional activity by the inhibition of PPAR-delta. The transcriptional modulators used are all multifunctional modulators that may not only achieve specification of their activity by nuclear receptor cross-talk [21-23] but may also have important receptor dependent (genomic and non-genomic) as well as independent (non-genomic) activities [17, 18, 24].
Furthermore, anti-inflammatory approaches were selected according to known effects of dexamethasone in hormonerefractory prostate cancer and interferon-alpha (at high-doses) in metastatic renal cell carcinoma. Interferon-alpha was used at a dose level for angiostatic activity, i.e. at very low doses.

In metastatic RCCC, we selected in a second consecutive trial an anti-inflammatory approach with presumably enhanced anti-inflammatory capacity: pioglitazone, coxib, and additionally interferon-alpha [12]. In HRPC, a combination of two activators of nuclear transcription factors (pioglitazone and dexamethasone) has been introduced [14].

A randomized phase II trial (metastatic melanoma) evaluated the additional effects of anti-inflammatory therapy in addition to metronomic low-dose chemotherapy on progression-free and overall survival (combined antiinflammatory/angiostatic vs. angiostatic approach) [9].

In the trials melanoma I, sarcoma I, and vascular sarcomas, we introduced a 14 day lead-in phase with antiinflammatory therapy only (pioglitazone plus rofecoxib or etoricoxib) $[8,9]$.

\section{Angiostatic Therapies}

Angiostatic therapy consisted of metronomic low-dose chemotherapy, either $50 \mathrm{mg}$ oral trofosfamide (Baxter) administered continuously 2 or 3 times daily or $1 \mathrm{~g} / \mathrm{m}^{2}$ to $1 \mathrm{~g}$ absolute oral capecitabine (Roche) administered twice per day. 


\section{Treatment Schedules}

Patients were centrally randomized for the Melanoma II trial. Arm A received $50 \mathrm{mg}$ oral trofosfamide (Baxter) administered continuously three times daily from day $1+$, Arm B of trofosfamide in the same dosage plus continuously $60 \mathrm{mg}$ oral pioglitazone (Takeda) and $25 \mathrm{mg}$ oral rofecoxib (MSD) once daily starting with day $1+$. Treatment was continued until disease progression was documented or for a maximum of six weeks after confirmation of CR. Following disease progression, a crossover from Arm A to B was allowed.

Patients treated in the vascular sarcoma trial (including one patient with Kaposi sarcoma), in the Melanoma I, Sarcoma I, and Langerhans cell histiocytosis trials received Arm B-therapy as described above. Melanoma and sarcoma patients had a lead-in phase with anti-inflammatory therapy alone over 14 days. Patients in the RCCC study I received $1 \mathrm{~g} / \mathrm{m}^{2}$ oral capecitabine (Roche) administered twice daily from day $1+, 60 \mathrm{mg}$ oral pioglitazone (Takeda). Patients enrolled before November 2004 also received $25 \mathrm{mg}$ oral rofecoxib daily, whereas patients enrolled after November 2004 were given $60 \mathrm{mg}$ oral etoricoxib daily instead, starting with day $1+$. Patients in study II (RCCC II) received additionally 4.5 MU IFN-alpha subcutaneously, three times per week, from day $1+$. Patients with cholangiocellular carcinoma were treated with the schedule of RCCC I.

\section{Combined Targeting of Wound Healing Processes}

In all studies, we selected transcriptional modulators including those of nuclear transcription factors with the aim to control tumor-associated inflammation. For metastatic melanoma, we performed a randomized phase II trial to directly study the impact of inflammation control on progression-free and overall survival. A historic comparison (RCCC I/II) shows the impact of weak versus strong control of tumor-associated inflammation on progression-free and overall survival in renal clear cell carcinoma. In hormone-refractory prostate cancer, published data from metronomic cyclophosphamide and dexamethasone treatment are available for a historical comparison [25].

Pre-treatment Evaluation is Indicated in the Respective Publications

\section{Evaluation of Efficacy}

Response and toxicity were evaluated in patients who had a follow-up duration of $\geq$ three weeks. Objective tumor responses were identified using the World Health Organization (WHO) criteria (vascular sarcomas, sarcomas, melanomas) or RECIST criteria (RCCC and HRPC).
Modulation of Tumor-associated Disease Traits

ECOG status ECOG performance status was routinely monitored.

Monitoring of CRP Serum CRP levels were measured in follow-up to evaluate the incidence of systemic inflammatory response in metastatic tumors dependent on the tumor histology and to determine the intensity of the inflammatory response as well as the time of inflammation response in relation to objective tumor response.

As part of an exploratory retrospective analysis, PFS and OS were evaluated separately for two groups of patients: (1) CRP responder: Patients with normal range CRP levels throughout the first six weeks of treatment and patients with elevated CRP levels, who responded with an at least $30 \%$ decrease within the first six weeks of treatment (two consecutive measurements at least 14 days apart). (2) CRP non-responder: Patients with $\leq 30 \%$ decline or increasing CRP levels in two consecutive measurements 14 days apart within the first six weeks of treatment.

Patients receiving a lead-in phase with anti-inflammatory therapy were monitored for CRP at study inclusion and in a 14 day interval.

\section{Metastatic Sites}

On the background of the discussion, whether combined biomodulatory therapies have any tissue specificity, i.e. are dependent on the cellular tumor-stroma composition at an organ site, we analyzed the response dependent on the localization of the metastatic organ sites. To assess whether an anti-inflammatory/angiostatic treatment approach has any impact on the metastatic spread during progression, we analyzed the metastatic sites after progression on study medication.

\section{Statistics and Data Analysis}

Primary endpoints in all trials were PFS and treatment safety. Analysis of treatment safety was restricted to patients receiving study medication, analysis of the tumor response to patients who were treated for at least three weeks. The overall response rate was defined as percentage of patients with confirmed CR or PR. SD was defined as no tumor progression $(<25 \%)$ during a six months treatment interval. Response duration was calculated from randomization or study inclusion to the date of first observation of progressive disease (PD) or death. Progression-free survival was defined as the interval between the beginning of treatment and disease progression. Survival duration was calculated from randomization or study inclusion. Survival distributions were generated using the Kaplan-Meier 
method. Survival analyses were performed on eligible patients, the full analysis set (FAS) and on the intent-to treat (ITT) population (defined as all randomly assigned patients). In addition, the Fisher exact test and the "Student t"-test were used to identify significant associations between clinical and biologic variables.

\section{Results}

In total, 224 patients with metastatic cancer from eleven centers and various medical specialties including urology, dermatology, gastroenterology, and hematology/oncology were treated within seven trials: The intention was to show the efficacy and tolerability of a combined anti-inflammatory (pioglitazone plus coxib) and angiostatic therapy (trofosfamide or capecitabine) in advanced tumor stage and in a high number of refractory cancer (10 to 63\%). More detailed patient characteristics may be found in the respective publications [8-15].

All trials were initiated as palliative therapies. Therefore, it is remarkable that we could observe objective response (3 to $48 \%$ ) and continuous complete remissions independent of the tumor type (vascular sarcoma, RCCC, melanoma, hormone-refractory prostate cancer, cholangiocellular carcinoma, and Langerhans' cell histiocytosis) in all treatment groups (except RCCC I) (Table 2).

Median progression-free survival as the primary endpoint in all trials is listed in Table 3. Interestingly, despite of the inclusion of systemically pre-treated patients at a high percentage $(10-63 \%)$, the PFS rate is comparable to the respective rate achieved in first-line therapy (trial RCC II, Melanoma II, and cholangiocellular carcinoma). In metastatic melanoma (Melanoma II), metronomic low-dose chemotherapy with trofosfamide seems to be even equivalent to standard DTIC treatment in a historical comparison [26-28].
Tailored Modeling of Tumor-associated Disease Traits

Overall, five different tumor-associated disease traits were followed within each trial with biomodulation-derived biomarkers: (1) Changes in the ECOG status, in (2) serum CRP levels, (3) the resolution of paraneoplastic syndromes, (4) objective tumor response at single metastatic organ sites, and (5) the dissemination of metastatic disease at tumor progression (metastatic spread).

ECOG performance status ECOG performance status could be improved in all trials (19 to $100 \%$ ). As expected in the Melanoma II trial, no ECOG improvement was observed within treatment arm A (without anti-inflammatory therapy) (Table 4). An improvement of the performance status due to inflammation control was possible on the basis of a very low rate of grade III toxicities in all trials (Table 5).

Paraneoplastic syndromes The anti-inflammatory activity of the chosen treatment schedules was additionally shown by the resolution of paraneoplastic syndromes: Hypoglycemia and lupus erythematodes respectively [29, 30].

Serum CRP level in follow-up The incidence of elevated CRP levels $(>10 \mathrm{mg} / \mathrm{L})$ at study inclusion differed considerably between the different tumor types (Table 4). In groups with consistently elevated CRP levels (RCCC, melanoma, sarcoma, Langerhans' cell histiocytosis), a significant CRP response $(>30 \%)$ was observed during the lead-in phase with anti-inflammatory therapy alone or during 4 to 6 weeks of combined treatment. Thus, efficacy of an anti-inflammatory therapy could be sufficiently followed in metastatic diseases with constitutive systemic inflammatory response (Table 4).

A CRP response indicated stable disease or objective response in most patients; however, few patients experi-
Table 2 Combined targeting of angiogenesis and inflammation: efficacy

\begin{tabular}{|c|c|c|c|c|}
\hline \multirow[t]{2}{*}{ Tumor type } & \multicolumn{4}{|l|}{ Response } \\
\hline & No. of patients & $\begin{array}{l}\text { Partial } \\
\text { response \% }\end{array}$ & $\begin{array}{l}\text { Complete } \\
\text { response \% }\end{array}$ & $\mathrm{cCR} \%$ \\
\hline Sarcomas I & 21 & 19 & 16 & 5 \\
\hline Angiosarcomas & 6 & 17 & 33 & 17 \\
\hline Melanoma I & 19 & 10 & 5 & 0 \\
\hline Melanoma II Arm B & 35 & 9 & 3 & 3 \\
\hline Langerhans' cell histiocytosis & 2 & - & 100 & 100 \\
\hline $\begin{array}{l}\text { Renal clear cell carcinoma I } \\
\text { (no IFN-a) }\end{array}$ & 18 & 0 & 0 & 0 \\
\hline $\begin{array}{l}\text { Renal clear cell carinoma II } \\
\text { (plus IFN-a) }\end{array}$ & 33 & 35 & 13 & 6 \\
\hline Hormone-refractory prostate cancer & 36 & 28 & 6 & 6 \\
\hline Cholangiocellular carcinoma & 21 & 24 & 5 & 5 \\
\hline
\end{tabular}


Table 3 Progression-free/overall survival with combined angiostatic plus anti-inflammatory therapy

\begin{tabular}{|c|c|c|c|c|c|}
\hline \multirow[t]{2}{*}{ Trial } & \multicolumn{2}{|l|}{ Treatment } & \multicolumn{3}{|c|}{ Median Progression-free/overall survival (months) } \\
\hline & Angiostatic & Anti-inflammatory & $\%$ pretreated patients & Trial & Historical control (first- line) \\
\hline RCCC I & Capecitabine & Pio/Rofe & 39 & $4.7 / 16.2$ & \\
\hline RCCC II & Capecitabine & Pio/Eto/IFN- $\alpha$ & 21 & $11.5 / 25.6$ & 11.0/n.a.(for sunitinib) \\
\hline HRPC & Capecitabine & Pio/Eto/Dexa & 39 & $3.6 / 14.4$ & n. a./17.5 (for taxotere) \\
\hline \multicolumn{6}{|l|}{ Melanoma II } \\
\hline Arm A & Trofosfamide & - & 63 & $1.2 / 8.2$ & n.a./5.6 (for DTIC) \\
\hline Arm B & Trofosfamide & Pio/Rofe & 60 & $2.0 / 18.8$ & \\
\hline Cholangiocellular carcinoma & Capecitabine & Pio/Rofe & 10 & $2.0 / 8.0$ & PR plus stable disease $20-73 \%$ \\
\hline
\end{tabular}

Pio pioglitazone, Rofe rofecoxib, Eto etoricoxib, RCCC renal clear cell carcinoma, HRPC hormone refractory prostate cancer, n.a. not available

enced progressive disease $(6 \%)$ despite of a CRP response. Therefore, CRP response indicates a tailored modeling of a tumor-associated disease trait but CRP assessment should not be used as a tumor marker. In HRPC, a CRP decrease was always paralleled by PSA response, whereas CRP response and/or ECOG improvement preceded objective responses by months ( 3.1 months to 8.6 months) in all other trials with the exception of individual patients with vascular sarcomas [8]. Due to the observed objective tumor responses to anti-inflammatory therapy in diseases without initial systemic inflammatory reaction such as HRPC, localized inflammatory tumor-associated processes have to be suggested as basis for the observed objective tumor responses [31].

Impact of anti-inflammatory therapy The efficacy of an anti-inflammatory therapy alone has already been shown in a randomized comparison in advanced cancer [32]. We can now extend the experiences on anti-inflammatory therapy: (1) Anti-inflammatory therapy adds further benefits to angiostatic low-dose chemotherapy by a significant improvement of OS in metastatic melanoma, although the objective response rates in both treatment arms did not significantly differ (randomized melanoma phase II trial, Melanoma II), and (2) the intensity of an anti-inflammatory approach, as indicated by the extent of CRP decrease in serum, may have significant impact on outcome (sequentially performed RCC trials I/II).

Intensification of anti-inflammatory therapy Two kinds of intensification of anti-inflammation were tested including a second transcriptional modulator, i.e. dexamethasone (HRPC) or interferon-alpha (RCCC II) (Table 1). The addition of low dose interferon-alpha to pioglitazone and COX-2 inhibitor dramatically increased the control of tumor-associated inflammation and consecutively improved

Table 4 Tumor-associated inflammation in metastatic cancer

\begin{tabular}{|c|c|c|c|c|c|}
\hline Trial & $\begin{array}{l}\text { Frequency of CRP } \\
\text { elevation }>10 \mathrm{mg} / \mathrm{L} \\
(\%)\end{array}$ & $\begin{array}{l}\mathrm{CRP}>30 \% \\
\text { response } \\
(\% \text { patients })\end{array}$ & $\begin{array}{l}\text { Significance of CRP } \\
\text { response during } 2 \text { to } \\
6 \text { weeks on treatment }\end{array}$ & $\begin{array}{l}\text { Improvement of } \\
\text { ECOG status \% } \\
\text { patients }\end{array}$ & $\begin{array}{l}\text { Progression-free survival } \\
\text { and overall survival }\end{array}$ \\
\hline RenaI clear cell carcinoma I & 72 & 69 & $\mathrm{p}=0.32$ & 22 & \multirow{4}{*}{$\begin{array}{l}\text { Significant improvement } \\
\text { of PFS and OS in RCCC II } \\
\text { (non randomized) }\end{array}$} \\
\hline Renal clear cell carcinoma II & 100 & 100 & $\mathrm{p}=0.0005$ & 24 & \\
\hline Hormone-refractory prostate cancer* & 28 & 11 & $\mathrm{p}=0.67$ & 30 & \\
\hline Melanoma I & 81 & 88 & $\mathrm{p}=0.004$ & 19 & \\
\hline \multicolumn{6}{|l|}{ Melanoma II } \\
\hline Arm A & 87 & 6 & $\mathrm{p}=0.52$ & 0 & \multirow{5}{*}{$\begin{array}{l}\text { Significant improvement } \\
\text { of overall survival } \\
\text { (CRP responder) }\end{array}$} \\
\hline Arm B (randomized) & 100 & 69 & $\mathrm{p}=0.0007$ & 27 & \\
\hline Sarcoma & 79 & 74 & $\mathrm{p}=0.006$ & 28 & \\
\hline Angiosarcoma * & 100 & 100 & - & - & \\
\hline Langerhans' cell histiocytosis & 100 & 100 & - & 100 & \\
\hline
\end{tabular}

*Resolution of paraneoplastic syndromes: lupus erythematodes, hypoglycaemia 
Table 5 Toxicities WHO Grade 3 (no Grade 4 toxicities) within all seven trials ( $n=224$ patients)

\begin{tabular}{lcll}
\hline Toxicity & No. of patients (\%) & Trial & Toxicity related to the following drug \\
\hline Cushing syndrome & $1(0.4)$ & HRPC & Dexamethasone \\
Depression & $1(0.4)$ & RCCC & Interferon-alpha \\
Hand-Foot-Syndrome & $5(2.2)$ & CCC, HRPC & Capecitabine \\
Hematotoxicity & $14(6.2)$ & All trials & Metronomic chemotherapy \\
Edema & $5(2.2)$ & All trials & COX-2 inhibitor \\
Nausea/Vomiting & $3(1.3)$ & All trials & - \\
\hline
\end{tabular}

$\mathrm{CCC}=$ cholangiocellular carcinoma, $\mathrm{RCCC}=$ renal clear cell carcinoma, $\mathrm{HRPC}=$ hormone refractory prostate cancer

the tumor response as well as the survival rate (historical comparison). These results demonstrate that strong inflammation control may be an important prerequisite for the response in metastatic, non-resectable RCCC. In HRPC, dexamethasone showed very modest anti-tumor activity. However, the addition of a glitazone (plus coxib) resulted in a high response rate, interestingly even up to the achievement of complete remission. Due to the poor monoactivity of capecitabine in HRPC, most activity of the schedule might be related to the anti-inflammatory approach.

Combined transcriptional modulation The combined use of transcription modulators for inflammation control in HRPC (dexamethasone, pioglitazone, and coxib) and in RCCC II (interferon-alpha, pioglitazone, and coxib) - and glitazones plus coxib in all the other tumor types - seems to improve outcome in comparison to historical controls or is at least equivalent but with less therapy-related toxicity. Except for the monoactivity of metronomic low-dose chemotherapy in advanced melanoma and presumably in angiosarcomas, all other treatment components, i.e. interferon-alpha at very low dose-levels, pioglitazone, coxibs, and low-dose dexamethasone have very modest or none mono-activity at all in the respective tumor types [33-38]. Exclusively their combination paves the way for objective responses via transcriptional cross-talks.

Angiostatic therapy Metronomic low-dose chemotherapy showed a significant activity in the randomized melanoma trial (melanoma II) (Table 2). Recently published data disclosed that the second drug capecitabine has a rather modest activity in HRPC [33] when administered in a nearly metronomic manner. Thus, most clinical effects in HRPC may be related to a combined anti-inflammatory activity. This observation is supported by unpublished data indicating objective responses after a change to metronomic low-dose treosulfan (250 mg twice daily) in patients with progressive HRPC on study medication. In cholangiocellular carcinoma, anti-inflammatory and angiostatic effects cannot be separated and assessed in correlation to historical data.
Metastatic sites and response To evaluate tumor-stromaspecific activities of the administered drugs, we studied whether specific single metastatic organ sites respond predominantly to combined biomodulatory therapy. An organ-specificity of combined anti-inflammatory and angiostatic activity could be observed in HRPC: in bone lesions, resolution or $>50$ regression (scintigraphy) of metastatic lesions could be observed, whereas only minor responses or stable diseases were diagnosed in all other metastatically involved organs.

Metastatic sites at progression Overall, $76 \%$ of the patients within the Melanoma trial II, RCCC trial II, and HRPC trial were systematically studied for metastatic sites at tumor progression. Interestingly, $67 \%$ of these patients had no additional metastatic organ sites at the time of progression, but local tumor progression or additional metastasis in the organ involved originally. This finding could indicate an attenuation of metastatic spread by the combined antiinflammatory and angiostatic approach. Probably because of the short median progression-free survival in Melanoma II, no significant differences could be found between the two treatment arms concerning metastatic spread at progression.

The treatment and response characteristics support biomodulatory mechanisms of action: (1) No or poor single agent activity of each administered drug, (2) a very moderate toxicity profile during long-term drug administration up to 26 months, (3) very delayed objective responses, (4) improved overall survival without an increase of response rate (randomized Melanoma trial), (5) significant modulation of tumor-associated disease traits, e.g. inflammation, ECOG status, paraneoplastic syndromes, (6) activity depending on the metastatic organ site in HRPC, and (7) predominant site of progression at the original localization of the metastases.

\section{Safety Profile}

The toxicity profiles of the presented biomodulatory approaches are modest as reflected in a low rate of WHO grade $>2$ toxicities and no grade 4 toxicities (Table 6). Thus, the desirable therapeutic effects could be achieved by 
Table 6 Combined targeting of angiogenesis and inflammation: patients with progressive disease

\begin{tabular}{lclc}
\hline Tumor type & No. of patients & Targeted (nuclear) transcription factors & Progressive disease No. of patients (\%) \\
\hline Sarcomas I & 21 & PPAR $\alpha / \gamma$, PPAR $\delta$ & $4(19)$ \\
Angiosarcoma & 6 & PPAR $\alpha / \gamma$, PPAR $\delta$ & 0 \\
Melanoma I & 19 & PPAR $\alpha / \gamma$, PPAR $\delta$ & $4(21)$ \\
Melanoma II Arm B & 35 & PPAR $\alpha / \gamma$, PPAR $\delta$ & $6(17)$ \\
Langerhans' cell histiocytosis & 2 & PPAR $\alpha / \gamma$, PPAR $\delta$ & 0 \\
Renal clear cell carcinoma I & 18 & PPAR $\alpha / \gamma$, PPAR $\delta$ & $9(50)$ \\
Renal clear cell carcinoma II & 33 & PPAR $\alpha / \gamma$ PPAR $\delta$ via IFN- $\alpha$ receptor & $2(7)$ \\
Hormone-refractory prostate cancer & 36 & PPAR $\alpha / \gamma$, PPAR $\delta$ glucocorticoid receptor & $5(14)$ \\
Cholangiocellular carcinoma & 21 & PPAR $\alpha / \gamma$, PPAR $\delta$ & 0 \\
\hline
\end{tabular}

Receptor ligands: PPAR $\alpha / \gamma$ agonist, PPAR $\delta$ antagonist (COX-2 inhibitor), dexamethasone, interferon-alpha PPAR peroxisome proliferator-activated receptor

minimizing side effects, even by improving the ECOG status before objective tumor response will be achieved. Because of the low rate of grade 3 toxicities, long-term drug administration up to more than two years was possible (median time on study medication 3.6 months (range 0.5 to 26.0). The low rate of toxicities $>$ grade 2 might be related to the fact that each drug is not administered at a maximal tolerable dose, even not at a dose level where mono-activity may be observed.

A second important point for safety evaluation is the question whether activating biomodulators may promote tumor activity. The stimulatory therapy with transcriptional modulators (interferon-alpha, PPAR-alpha/gamma agonist, dexamethasone) did obviously not enhance the percentage of patients with continuously progressive disease compared to standard therapies in the individual tumor types.

\section{Discussion}

The uniform treatment schedules presented were initially chosen to facilitate disease stabilization in patients with advanced and pre-treated cancer with less toxic agents. Surprisingly, it turned out that these treatment schedules have the capacity to induce objective responses (3 to $48 \%$ ) and, in individual patients, even continuous complete remissions in every tumor type mentioned. Furthermore, they may induce OS rates, which compare with established standard first-line therapies.

With respect to the multi-facetted activities of the administered drugs (anti-proliferative, angiostatic, antiinflammatory, metabolic activity, immunomodulatory), and their differential cell-specific activities, the exact mechanisms of action of the selected drug combinations are difficult to pin down $[17,18,39]$. The studied drug combinations are interacting with the systems biology of the different cell types at regulatory sites and have both genomic and non-genomic activity.
With the exception of individual patients suffering from vascular sarcoma, responses to therapy occurred very delayed and three phases were observed: (1) Inhibition of further tumor progression, (2) prolonged disease stabilization by 3.1 months to a mean of 8.6 months, followed by (3) objective responses. In some tumor types, response to therapy could be monitored by a serum parameter, Creactive protein, indicating the tailored modeling of a tumor-associated disease trait, namely inflammation. Systemic tumor-associated inflammation, however, was no prerequisite for objective tumor response to a combined anti-inflammatory therapy approach as shown in hormone refractory prostate cancer that has a very low incidence of systemic inflammatory events [31].

On the basis of these observations, we now postulate tumor-associated inflammation as both a pathophysiologically important element and a therapeutic target but without presupposing causal relationships between inflammation and tumor progression. On the contrary, the prerequisites for our clinical observations, i.e. the multifacetted regulatory activities of the single administered drugs and the differential responses of the multiple cell types within the tumor compartment, reveal the relations of conditioned and conditioning tumor-promoting moments as reciprocal on the basis of pathophysiologically important interacting elements (e.g. inflammation, angiogenesis, and tumor cell proliferation). The still 'indistinct' but regulatory activity profile of the administered drugs and the favorable therapy results of the uniform treatment concept in a broad variety of different tumor types strongly support our hypothesis that tumor growth may be successfully attenuated by targeting the tumor system's biology simultaneously at multiple regulatory sites, e.g. (nuclear) transcription factors.

Pathologic systems biological processes in cancer may be reported from different observation levels: (1) In Loewenstein's view pathologic cancer processes are predominantly mirrored in a deficient cell-cell communication 
[40]. (2) The initial source of observation may also be an altered systems-associated cell composition, and (3) distorted functions of single cell systems within the tumor microenvironment $[1,3,6]$. Inflammatory processes have been identified to be involved in tumor systems biology independently of the viewpoint of observation.

One aspect is getting of growing systems-therapeutic interest since normal adult and cancer stem cells may be detected by selective expression of the transcription factor Okt4 [41, 42]: Inflammation plays a critical role on all virtual stages of tumor development, tumor initiation, promotion and progression [43]. The inhibition of gap junctional communication has been identified as an important mechanism by which inflammatory processes affect cancer development: Cancer cells exist in two forms, those that do not express connexin (gap junction genes), and those that express connexin genes but that gap junction function has been rendered non-functional by oncogenes/loss of tumor suppressor genes [44]. Here the use of agents to turn on critical genes, i.e., such as the connexin genes seems to be important [4547]. That the cancer stem cell must be promoted by a number of inflammatory conditions, particularly in the metastatic stage of cancer disease (cachexia!) fits with the successful use of anti-inflammatory therapy components in the present systems-targeted treatment strategy [48].

Conventional therapy methods commonly neglect the complexity of the tumor compartment. They mainly target the molecular-genetically highly variable tumor cell, whose variability is explained by the complexity of the tumor development. By blocking a pathological signaling pathway with a small molecule or an antibody, the whole tumor system should be destroyed, synonymously with the assumption that tumor development could result from a single causative principle. Furthermore, combining cytotoxic therapy elements guided by the simple availability of drugs buys moderately enhanced efficacy at a simultaneously enhanced toxicity profile, as shown by many studies.

A lead back to a final first principle that may be therapeutically targeted to eradicate metastatic cancer is generally not permitted, in particular in knowledge of the multi-facetted activity profile of the administered biomodulatory agents. However, instead of such a lead back to a first principle, we have to deal with multiple and various constellations of elements (aggregated action effects), one of which - in our case - is tumor-associated inflammation. The constellation of elements has to be broken down to its single moments, but, simultaneously, we have to understand the relationship between one another rather than separately adding one to another and thereby neglecting the importance within the complex constellation. The principle therapeutic difficulty lies in this point.

The therapeutic components chosen directly address this difficulty based on the hypothesis that the combined activity of regulatory but pleiotropic agents, particularly transcription modulators (besides the angiostatic approach), may shape the tumor's organization, e.g. the 'wound healing' mechanisms, by attenuating simultaneously multiple activities involved in tumor growth such as angiogenesis, anti-inflammation, and proliferation. This hypothesis is supported by seven treatment-related characteristics: (1) No or poor single agent activity of each administered drug (predominantly combined regulatory activity) when given alone, (2) a very moderate toxicity profile during long-term drug administration (presumably no dose-response relationship), (3) very delayed objective responses (stable shaping and focusing of the tumor system's organization), (4) improved overall survival without an increase of the response rate in arm B of the randomized Melanoma II trial (biomodulatory activity), (5) significant modulation of tumor-associated disease traits, e.g. inflammation, ECOG status, paraneoplastic syndromes (biomodulation-derived biomarkers), (6) activity depending on the metastatic organ site in HRPC (tumor-stroma-specificity as expected from the known differential behavior of the various cell types within the tumor compartment, and the varying stroma cell compositions at the different metastatic sites), and (7) predominant site of progression at the original localization of the metastases (hints for impact on metastatic processes). Preclinical data on the action of COX-2 inhibitors and PPAR-alpha agonists are already revealing antimetastatic activity $[49,50]$.

Even if metronomic chemotherapy has any cytotoxic activity in the classic sense, the response characteristics do not support a response behavior as usually found in response to pulsed chemotherapy.

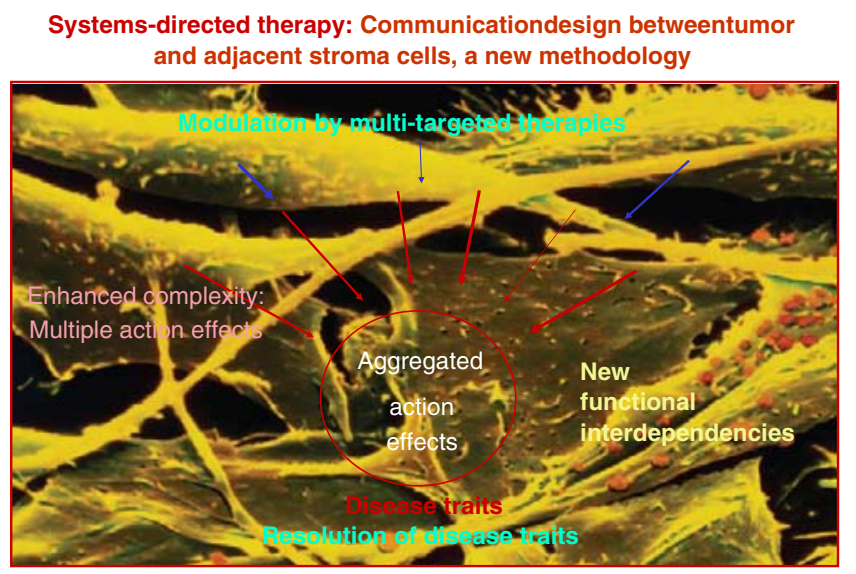

Fig. 1 The differential response patterns within our clinical trials indicate the therapies' systems biological activity. Understanding systems biology as adjustable size may break through the barrier of complex tumor-stroma-interactions in a therapeutically relevant way: Comparatively high efficacy at moderate toxicity. Structured systemsdirected therapies in metastatic cancer may get a source for detecting tumor-associated complex aggregated action effects as adjustable sizes available for targeted biomodulatory therapies 
The clinical efficacy of the combined anti-inflammatory and angiostatic approach in different tumor types reveals preserved regulatory elements for targeting 'wound healing' processes with transcriptional regulators (biomodulatory agents) in tumor and adjacent stroma cells. (1) The favorable clinical results achieved with a small repertoire of transcriptional modulators indicate a constitutive dysregulation of distinct transcription factors, which - on the other hand - seems to be paradoxically linked to the heterogeneous tumor-associated molecular-genetic aberrations depending on the tumor type [51]. (2) The combined genomic/non-genomic therapy approach specifically shapes the organization of the tumor-stroma-interaction. (3) The clinically combined activity of (nuclear) transcription factors in the RCCC II and HRPC trial give sufficient clinical evidence for a crosstalk between drug-activated/ deactivated transcription factors.

The focus on the systems biology of a tumor as the original target of cancer therapy necessitates biomarkers that indicate stable response in the field of tumor-associated disease traits or tumor-associated phenomena such as inflammation, angiogenesis, coagulation, and metabolism. Rather than the primary or "classic" markers for tumor response including tumor shrinkage or decrease of tumor markers, this new group of markers reflects efficacious biomodulation. However, we are aware of the limitation that some of these tumor-associated phenomena mirroring tumor biomodulation are sometimes difficult to follow on a systemic level. They can not be uniformly interpreted across tumor entities as demonstrated in our example of HRPC in comparison to other tumors, when inflammation seems to be quite differently integrated in the tumors' pathophysiology: PSA decline was paralleled but not preceded by a CRP decline in HRPC, whereas in other tumor types including RCCC decrease of CRP or ECOG performance improvement preceded tumor response.

In the immediate presence and future, biomodulatory therapy approaches of metastatic tumors could be methodological tools of individualized tumor therapy: In contrast to 'causal' therapy approaches aiming at blocking aberrant tumor-associated pathways by a restricted repertoire of highly specific drugs, multiple potential modulators (activators and deactivators) of transcriptional processes are available for biomodulatory therapy approaches. According to our experiences, mono-activity of a single transcription modulator is no prerequisite for its successful use and their combined administration activity could be followed by respective biomarkers. Close monitoring would further allow us to choose other modulator combinations in cases of weak interactivity to facilitate objective tumor response.

Finally, the constitutive dysregulation of transcriptional activity is shown to be an important target for biomodulatory therapy approaches in metastatic cancer. Biomodula- tion in metastatic tumors provides tools for recognizing patterns in therapy-associated events via biomodulationderived biomarkers. Thereby, it enables (1) the shaping of the tumor system's organization and (2) the uncovering of endogenous sources such as transcription factors and their crosstalks for managing growth behavior by counterbalancing the tumor systems' biology.

Our seven published phase II trials on combined targeted therapy of tumor-associated wound healing mechanisms, e.g. inflammation and neoangiogenesis, have shown that using an approach for understanding systems biology as adjustable size, we may break through the barrier of complexity of tumor-stroma-interactions in a therapeutically relevant way (Fig. 1). For a targeted modulation, elements such as inflammation and neoangiogenesis are available, which are dysregulated on the basis of acquired chromosomal aberrations. Biomodulation of systems biological processes facilitate comparatively high efficacy at moderate toxicity.

General interpretations of the tumor's systems biology may not be performed in context-free explanations. The requirements of application (therapy schedule, tumor type) and the number of surrogate markers define the way the interpretation is conducted. Additionally, they define the hermeneutic understanding of extremely complex cellular interactions correspondingly to the chosen picture, the wound healing mechanisms. In the present case, this means the following: Naturally, the administered drugs, particularly the transcriptionally active modulators, have still an insufficiently illuminated spectrum of activities, which may be even dependent on the cell type. General interpretations concerning the systems biology do not obey the same categories of refutation as general theories and remain per se open for discussion. The logic of an explanation of the tumor's systems biology is the result of a connection between a hermeneutic understanding (wound healing mechanisms) and the causal explanation (e.g. co-regulatory activity of transcription factors).

Open Access This article is distributed under the terms of the Creative Commons Attribution Noncommercial License which permits any noncommercial use, distribution, and reproduction in any medium, provided the original author(s) and source are credited.

\section{References}

1. Galon J, Costes A, Sanchez-Cabo F et al (2006) Type, density, and location of immune cells within human colorectal tumors predict clinical outcome. Science 313:1960-1964

2. Dvorak HF (1986) Tumors: wounds that do not heal. Similarities between tumor stroma generation and wound healing. N Engl $\mathrm{J}$ Med 315:1650-1659 
3. Balkwill F, Coussens LM (2004) Cancer: an inflammatory link. Nature 431:405-406

4. Brahimi-Horn MC, Chiche J, Pouyssegur J (2007) Hypoxia signalling controls metabolic demand. Curr Opin Cell Biol 19:223-229

5. Dvorak HF (1987) Thrombosis and cancer. Hum Pathol 18:275-284

6. Mueller MM, Fusenig NE (2004) Friends or foes - bipolar effects of the tumour stroma in cancer. Nat Rev Cancer 4:839-849

7. Angelo LS, Kurzrock R (2007) Vascular endothelial growth factor and its relationship to inflammatory mediators. Clin Cancer Res $13: 2825-2830$

8. Vogt T, Hafner C, Bross K et al (2003) Antiangiogenetic therapy with pioglitazone, rofecoxib, and metronomic trofosfamide in patients with advanced malignant vascular tumors. Cancer 98:2251-2256

9. Reichle A, Bross K, Vogt T et al (2004) Pioglitazone and rofecoxib combined with angiostatically scheduled trofosfamide in the treatment of far-advanced melanoma and soft tissue sarcoma. Cancer 101:2247-2256

10. Reichle A, Klebl F, Bross K et al (2004) Pioglitazone and Rofecoxib combined with angiostatically scheduled capecitabine in far-advanced hepatobiliary carcinoma. Medimond S r 1, pp. 87-98

11. Reichle A, Vogt T, Kunz-Schughart L et al (2005) Antiinflammatory and angiostatic therapy in chemorefractory multisystem Langerhans' cell histiocytosis of adults. Br J Haematol 128:730-732

12. Reichle A, Grassinger J, Bross K et al (2006) C-reactive Protein in patients with metastatic clear cell renal carcinoma: an important biomarker for tumor-associated inflammation. Biomarker Insights 2:87-98

13. Reichle A, Vogt T, Coras B et al (2007) Targeted combined antiinflammatory and angiostatic therapy in advanced melanoma: a randomized phase II trial. Melanoma Res 17:360-364

14. Reichle A, Walter B, Berand A et al (2007) Induction of complete remission in metastatic hormone-refractory prostate cancer: a combined anti-inflammatory therapy approach. [abstract]. Journal of Clinical Oncology, 2007 ASCO Annual Meeting Proceedings, vol. 25 , no. 185

15. Coras B, Hafner C, Reichle A et al (2004) Antiangiogenic therapy with pioglitazone, rofecoxib, and trofosfamide in a patient with endemic kaposi sarcoma. Arch Dermatol 140:1504-1507

16. Kapainen A, Kieran MW, Huang S et al (2007) PPARalpha deficiency in inflammatory cells suppresses tumor growth. PLoS One 2:260e

17. Hafner C, Reichle A, Vogt T (2005) New indications for established drugs: combined tumor-stroma-targeted cancer therapy with PPARgamma agonists, COX-2 inhibitors, mTOR antagonists and metronomic chemotherapy. Curr Cancer Drug Targets 5:393419

18. Hafner C, Vogt T, Reichle A (2007) Conventional therapeutics with antiangiogenic activity. In: Davis DW, Herbst RS, Abbruzzese JL (eds) Antiangiogenic cancer therapy. CRC, Boca Raton, FL, pp 301-327

19. Liou JY, Ghelani D, Yeh S (2007) Non-steroidal anti-inflammatory drugs induce colorectal cancer cell apoptosis by suppressing 14-33epsilon. Cancer Res 67:3185-3191

20. Emmenegger U, Shaked Y, Maus S et al (2007) Pharmacodynamic and pharmacokinetic studies of low-dose metronomic cyclophosphamide in mice. Mol Cancer Ther 6:2280-2289

21. Ogawa S, Lozach J, Benner C et al (2005) Molecular determinants of crosstalk between nuclear receptors and toll-like receptors. Cell 122:707-721

22. Wang D, Wang H, Guo $Y$ et al (2006) Crosstalk between peroxisome proliferator-activated receptor delta and VEGF stimulates cancer progression. Proc Natl Acad Sci USA 103:1906919074
23. Leung KC (2004) Regulation of cytokine receptor signaling by nuclear hormone receptors: a new paradigm for receptor interaction. DNA Cell Biol 23:463-474

24. Akbiyik F, Ray DM, Gettings KF et al (2004) Human bone marrow megakaryocytes and platelets express PPARgamma, and PPARgamma agonists blunt platelet release of CD40 ligand and thromboxanes. Blood 104:1361-1368

25. Glode LM, Barqawi A, Crighton F et al (2003) Metronomic therapy with cyclophosphamide and dexamethasone for prostate carcinoma. Cancer 98:1643-1648

26. Tannock IF, de Wit R, Berry WR et al (2004) Docetaxel plus prednisone or mitoxantrone plus prednisone for advanced prostate cancer. N Engl J Med 351:1502-1512

27. Middleton MR, Grob JJ, Aaronson N et al (2000) Randomized phase III study of temozolomide versus dacarbazine in the treatment of patients with advanced metastatic malignant melanoma. J Clin Oncol 18:158-166

28. Motzer RJ, Hutson TE, Tomczak P et al (2007) Sunitinib versus interferon alfa in metastatic renal-cell carcinoma. N Engl J Med $356: 115-124$

29. Vogt T, Coras B, Hafner C, Landthaler M et al (2006) Antiangiogenic therapy in metastatic prostate carcinoma complicated by cutaneous lupus erythematodes. Lancet Oncol 7:695-697

30. Plikat K, Reichle A, Elmlinger MW et al (2003) Hypoglycemia associated with the production of insulin-like growth factor (IGF)-II by a hemangiopericytoma. Dtsch Med Wochenschr 128:257-260

31. McArdle PA, Mir K, Almushatat AS et al (2006) Systemic inflammatory response, prostate-specific antigen and survival in patients with metastatic prostate cancer. Urol Int 77:127-129

32. Lundholm K, Gelin J, Hyltander A et al (1994) Anti-inflammatory treatment may prolong survival in undernourished patients with metastatic solid tumors. Cancer Res 54:5602-5606

33. Morant R, Bernhard J, Dietrich D et al (2004) Capecitabine in hormone-resistant metastatic prostatic carcinoma - a phase II trial. Br J Cancer 90:1312-1317

34. Storlie JA, Buckner JC, Wiseman GA et al (1995) Prostate specific antigen levels and clinical response to low dose dexamethasone for hormone-refractory metastatic prostate carcinoma. Cancer 76:96-100

35. Porzsolt F, Messerer D, Hautmann R et al (1998) Treatment of advanced renal cell cancer with recombinant interferon alpha as a single agent and in combination with medroxyprogesterone acetate. A randomized multicenter trial. J Cancer Res Clin Oncol 114:95-100

36. Debrock G, Vanhentenrijk V, Sciot R et al (2003) A phase II trial with rosiglitazone in liposarcoma patients. Br J Cancer 89:1409-1412

37. Buckstein R, Kerbel RS, Shaked Y et al (2006) High-dose celecoxib and metronomic "low-dose" cyclophosphamide is an effective and safe therapy in patients with relapsed and refractory aggressive histology non-Hodgkin's lymphoma. Clin Cancer Res 12:5190-5198

38. Kopp HG, Kanz L, Hartmann JT (2006) Complete remission of relapsing high-grade angiosarcoma with single-agent metronomic trofosfamide. Anticancer Drugs 17:997-998

39. McCarty MF, Barroso-Aranda J, Contreras F (2008) PPARgamma agonists can be expected to potentiate the efficacy of metronomic chemotherapy through CD36 up-regulation. Med Hypotheses 70:419-23

40. Loewenstein WR, Kanno Y (1966) Intercellular communication and the control of growth: lack of communication between cancer cells. Nature 209:1248-1249

41. Tai MH, Chang CC, Kiupel M et al (2005) Oct-4 expression in adult human stem cells: evidence in support of the stem cell theory of carcinogenesis. Carcinogenesis 26:495-502

42. Webster JD, Yuzbasiyan-Gurkan V, Trosko JE et al (2007) Expression of the embryonic transcription factor Oct4 in canine 
neoplasms: a potential marker for stem cell subpopulations in neoplasis. Vet Pathol 44:893-900

43. Trosko JE, Tai MH (2006) Adult stem cell theory of the multistage, multi-mechanism theory of carcinogenesis: role of inflammation on the promotion of initiated cells. In: Dittmar T, Zaenker KS, Schmidt A (eds) Infections and inflammation: impacts on oncogenesis. (Contributions to Microbiology, Vol. 13. Infection and inflammation: impacts on oncogenesis). S. Karger AG, , Switzerland, pp 45-65

44. Trosko JE (2003) The role of stem cells and gap junctional intercellular communication in carcinogenesis. J Biochem Mol Biol 36:43-48

45. Ogawa T, Hayashi T, Tokunou M et al (2005) Suberoylanilide hydroxamic acid enhances gap junctional intercellular communication via acetylation of histone containing connexin43 gene locus. Cancer Res 65:9771-9778

46. Ruch RJ, Madhukar BV, Trosko JE et al (1993) Reversal of rasinduced inhibition of gap junctional intercellular communication, transformation, and tumorigenesis by lovastatin. Mol Carcinog 7:50-59

47. Trosko JE, Ruch RJ (2002) Gap junctions as therapeutic targets. Current Drug Targets 3:465-482

48. Trosko JE (2007) Gap junction intercellular communication as a 'Biological Rosetta Stone' in understanding, in a systems manner, stem cell behavior, mechanisms of epigenetic toxicology, chemoprevention and chemotherapy. J Membr Biol 218:93-100

49. Roche-Nagle G, Connolly EM, Eng M et al (2004) Antimetastatic activity of a cyclooxygenase-2 inhibitor. Br J Cancer 91:359-365

50. Grabacka M, Plonka PM, Urbanska K et al (2006) Peroxisome proliferator-activated receptor alpha activation decreases metastatic potential of melanoma cells in vitro via down-regulation of Akt. Clin Cancer Res 12:3028-3036

51. Shen H, Lentsch AB (2004) Progressive dysregulation of transcription factors NF-kappa B and STAT1 in prostate cancer cells causes proangiogenic production of $\mathrm{CXC}$ chemokines. Am J Physiol Cell Physiol 286:C840-C847 